\title{
Localized exciton dynamics in AlInGaN alloy
}

\author{
J.S. Huang ${ }^{\mathrm{a}, *}$, X. Dong ${ }^{\mathrm{a}}$, X.D. Luo ${ }^{\mathrm{a}}$, X.L. Liu ${ }^{\mathrm{a}}$, Z.Y. Xu ${ }^{\mathrm{a}}$, W.K. Ge ${ }^{\mathrm{b}}$ \\ anstitute of Semiconductors, Chinese Academy of Sciences, Beijing 100083, People's Republic of China \\ ${ }^{\mathrm{b}}$ Department of Physics, Hong Kong University of Science and Technology, Hong Kong, People's Republic of China
}

Received 15 October 2002; accepted 15 February 2003 by Z. Z. Gan

\begin{abstract}
Carrier recombination dynamics in AlInGaN alloy has been studied by photoluminescence (PL) and time-resolved PL (TRPL) at various temperatures. The fast red-shift of PL peak energy is observed and well fitted by a physical model considering the thermal activation and transfer processes. This result provides evidence for the exciton localization in the quantum dot (QD)-like potentials in our AlInGaN alloy. The TRPL signals are found to be described by a stretched exponential function of $\exp \left[(-t / \tau)^{\beta}\right]$, indicating the presence of a significant disorder in the material. The disorder is attributed to a randomly distributed QDs or clusters caused by indium fluctuations. By studying the dependence of the dispersive exponent $\beta$ on temperature and emission energy, we suggest that the exciton hopping dominate the diffusion of carriers localized in the disordered QDs. Furthermore, the localized states are found to have 0D density of states up to $250 \mathrm{~K}$, since the radiative lifetime remains almost unchanged with increasing temperature.
\end{abstract}

(C) 2003 Elsevier Science Ltd. All rights reserved.

PACS: 39.30. + w; 72.20.Jv; 73.50. $-\mathrm{h}$

Keywords: A. AlInGaN; A. Quantum dots; D. Hopping; D. Stretched-exponential decay

\section{Introduction}

Recently, Group-III nitride semiconductors have emerged as the leading material for the production of green-blue-ultraviolet (UV) light-emitting diodes (LEDs) and laser diodes (LDs). In particular, the quaternary AlInGaN material system has attracted a great deal of interest due to its potential applications for the energy band engineering and strain engineering [1-3]. The use of AlInGaN films as the barriers for InGaN quantum wells (QWs) was found to improve the luminescence properties by suppressing deep level emissions and changing the emission mechanism [4,5]. Furthermore, AlInGaN alloys were also reported being used as the active layers for multiple QWs in fabricating deep-UV LEDs with peak emission wavelengths from 305 to $340 \mathrm{~nm}$ [6-8]. In spite of

\footnotetext{
* Corresponding author. Address: P.O. Box 912 NLSM, Beijing 100083, China. Tel.: +86-10-82304870; fax: + 86-10-82305056.

E-mail address: hjs@red.semi.ac.cn (J.S. Huang).
}

the fast development in the device applications, however, the optical emission mechanism has not been well understood and there are some controversies in the reported papers. For instance, G. Tamulaitis et al. suggested that the luminescence comes from the transition of electrons between band edges in AlInGaN alloys [9]. While Mee-Yi Ryu et al. attributed the emission to the recombination of carriers/excitons localized at band-tail states [10, 11].

In this work, we have studied carrier recombination dynamics in AlInGaN alloys by photoluminescence (PL) and time-resolved PL (TRPL) at various temperatures from 14 to $250 \mathrm{~K}$. Our results demonstrate that the PL emissions result primarily from localized exciton recombination related to the quantum nanostructures such as quantum dots (QDs) in the alloy. The TRPL data clearly show the presence of a significant disorder in the material. The exciton hopping mechanism is suggested to be responsible for the observed non-exponential PL decay process. Furthermore, the localized states are found to have 0D density of states in our studied temperature range. 


\section{Experimental procedure}

The samples studied were grown by low-pressure metalorganic chemical-vapor deposition (MOCVD) on sapphire (0001) substrates. A low temperature $\left(550^{\circ} \mathrm{C}\right) \mathrm{GaN}$ buffer of $\sim 20 \mathrm{~nm}$ thickness was first deposited, followed by the deposition of $1 \mu \mathrm{m}$ of $\mathrm{GaN}$ grown at $1030^{\circ} \mathrm{C}$. A $0.5-\mu \mathrm{m}-$ thick AlInGaN layer was then grown on the GaN layer. The growth temperature of AlInGaN layers was in the range of $800-880{ }^{\circ} \mathrm{C}$. All other growth conditions were kept unchanged for different samples. The growth pressure was 76 Torr. In the growth, trimethylgallium (TMG), trimethylaluminum (TMAl), ethyldimethylindium (EDMIn) and ammonia were used as precursors and $\mathrm{SiH}_{4}$ was used for n-type doping. The detailed growth procedure can be found elsewhere [12]. The composition of the samples was determined by energy dispersive system (EDS) and X-ray diffraction (XRD). In this work, we use a typical sample of $\mathrm{Al}_{0.12} \mathrm{In}_{0.095} \mathrm{Ga}_{0.785} \mathrm{~N}$ as an example to study the carrier dynamics in this quaternary alloy system. For TRPL measurements, the PL was excited by frequency-doubled $(\lambda=360 \mathrm{~nm})$ laser pulses from a Ti/Sapphire mode-locked femtosecond laser and the time-correlated signals were analyzed by a two-dimensional (2D) synchronous streak camera with an overall resolution of better than $15 \mathrm{ps}$. PL measurement was performed in $\mathrm{cw}$ configuration using a combination of a cooled GaAs PMT with a Data-Link electronic system.

\section{Results and discussion}

We have first measured the PL spectra of the $\mathrm{Al}_{0.12-}$ $\mathrm{In}_{0.095} \mathrm{Ga}_{0.785} \mathrm{~N}$ sample at various temperatures. The spectral width is quite broad even at low temperature (shown in the inset of Fig. 1), indicating the existence of local potential fluctuations in the material. In Fig. 1, the temperature dependence of the PL peak energy is plotted. It can be seen that the peak energy variation with temperature does not follow the Varshni law for typical AlGaN and InGaN alloys $[13,14]$. For comparison, a typical temperature dependence of the peak energy is shown in the same figure by a dot line with the Varshini parameters $\alpha=0.832 \mathrm{meV} / \mathrm{K}$ and $\beta=$ $835.6 K$ [14]. This unusual temperature dependence has been reported previously and taken as a characteristic behavior for the self-organized QDs [15], where the transfer of thermally excited carriers to the energetically low-lying states leads to the fast red-shift of exciton emission. In the case of AlInGaN quaternary alloy, nanoscale fluctuations in the indium concentration are expected to be easily formed, as AlInGaN quaternary alloys were reported to be InGaNlike rather than $\mathrm{AlGaN}-$ like, even when $\mathrm{Al}$ composition is much higher than indium [16]. In fact, non-uniform distribution of compositions in SEM image was observed in our AlInGaN sample (not shown). Therefore we believe

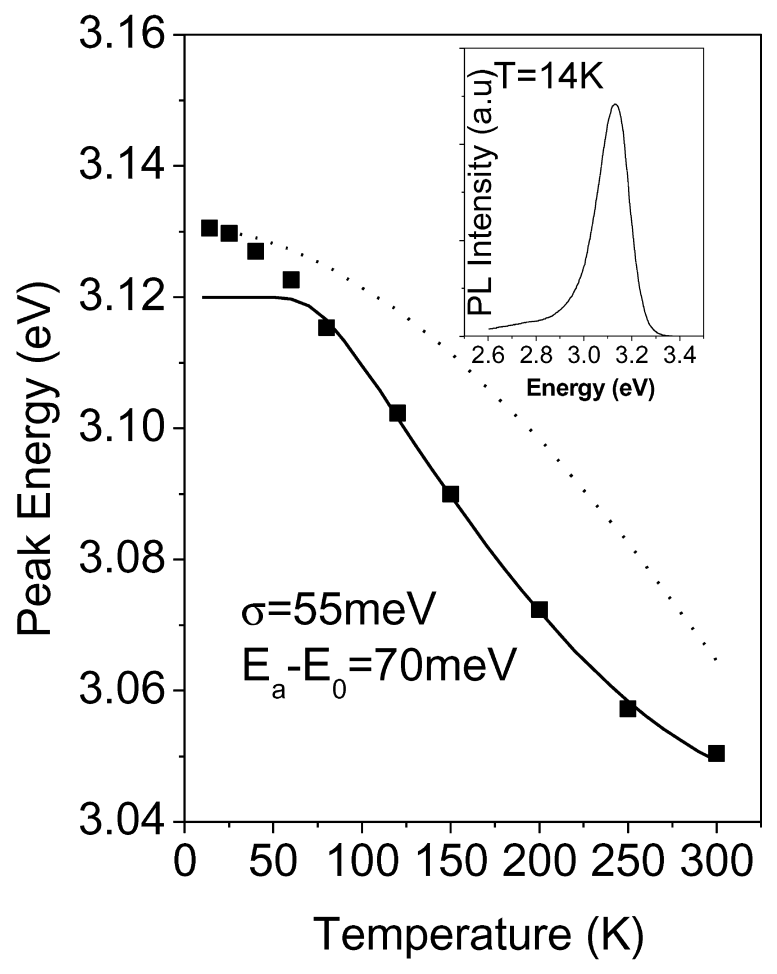

Fig. 1. Temperature dependence of the PL peak energy. Solid squares are the experimental data and the solid line is the fitting result. The inset is the PL of the sample at $14 \mathrm{~K}$.

that there exists a randomly distributed QD-like nanostructures in our AlInGaN sample as often reported in InGaN system $[17,18]$. The formation of QDs in $\mathrm{InGaN}$ is mainly due to the phase separation. The reported sizes of such kind of QDs is in the range of $0.6-3 \mathrm{~nm}$, much smaller than $10-$ $40 \mathrm{~nm}$ in self-organized InGaN QDs grown in StranskiKrastanow growth mode, and the QDs density can be as high as $5 \times 10^{11} \mathrm{~cm}^{-2}$. Then it is expected that the coupling between individual QDs and the transfer of carriers by tunneling will be greatly enhanced, resulting in the fast redshift of the exciton energy with temperature. Assuming the tunneling effect is analogous to thermal transfer process, we employed the thermal transfer model $[19,20]$ to calculate the temperature dependence of peak energy in our sample. Here the distribution of energy for QDs is assumed to be a Gaussian-like, that is, $\rho(E) \propto \exp \left[-\left(E-E_{0}\right)^{2} / 2 \sigma^{2}\right.$, where $\sigma$ and $E_{0}$ present the width and center of the distribution function, respectively. The peak energy $E(T)$ can be obtained using the equation

$E(T)=E_{0}-x(T) k_{B} T$,

and $x$ can be obtained by numerically solving the following equation:

$x \mathrm{e}^{x}=\left(\frac{\tau_{\mathrm{r}}}{\tau_{\mathrm{nr}}}\right)\left[\left(\frac{\sigma}{k_{B} T}\right)^{2}-x\right] \mathrm{e}^{\left(E_{0}-E_{\mathrm{a}}\right) / k_{B} T}$, 
where $\tau_{\text {tr }}$ is the transfer time; $\tau_{\mathrm{r}}$ is the carrier recombination time; $E_{\mathrm{a}}$ represents a barrier level that carriers must overcome to transfer. The calculated result is shown in Fig. 1 as a solid line. To our surprising, the fitting between the calculation and the experimental data is very well when $T>50 \mathrm{~K}$ with a fitting parameter $E_{\mathrm{a}}-E_{0}=70 \mathrm{meV}$. This energy difference reflects the magnitude of the carrier localization. The measured large localization energy is consistent with our hypothesis that the emission comes from the recombination of excitons localized in QDs. On the other hand, the AlInGaN alloy can also be regarded as a disordering material system due to the random distribution in the size and/or composition of the QDs. This point of view has been further demonstrated in our TRPL measurements.

The TRPL measurements of the sample were carried out in the temperature range from 14 to $250 \mathrm{~K}$. Fig. 2(a) shows PL decay curves at three typical temperatures. It is found that the decay process is characterized by a non-exponential function at all temperatures, and this non-exponential decay can be well described by a stretched exponential function, as analyzed by Chen et al. [21] for disordered low-dimensional semiconductors:

$I(t)=I_{0} \exp \left[-\left(\frac{t}{\tau}\right)^{\beta}\right]$,

where $I(t)$ is the PL intensity at time $t$ and $I_{0}$ is $I(0) ; \beta$ is a dispersive exponent and $\tau$ is the lifetime. This decay law is often encountered in the disordered systems and considered as a consequence of the dispersive diffusion of the photoexcited carriers. It has been successfully applied to explain the optical properties in InGaN QDs [22,23] and porous silicon [24,25]. In general, carrier dispersive diffusion among different spatial regions can be due to the excitation of carriers from localized to extended states
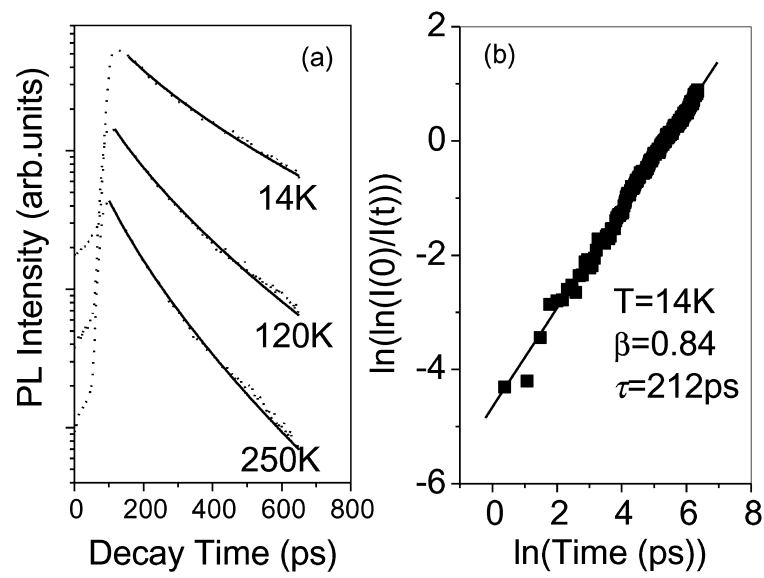

Fig. 2. (a) TRPL decay curves of the $\mathrm{Al}_{0.12} \mathrm{In}_{0.095} \mathrm{Ga}_{0.785} \mathrm{~N}$ sample at 14, 120 and $250 \mathrm{~K}$, respectively. The dots lines are experimental data and the full lines are the fitting using Eq. (3) (b) $\ln [\ln (I(0) / I(t))]$ versus $\ln$ (time). The obtained dispersive exponent $\beta$ and $\tau$ are indicated in the figure. (energetic disorder), or due to the hopping (tunneling) of electrons among localized states (topological disorder). In AlInGaN, fluctuations in the alloy composition, especially the fluctuations in indium concentration can produce both energetic and topological disorder. The theory suggest that a temperature-dependent $\beta$ is associated with a distribution of activation energies, whereas a constant $\beta$ arises from a dispersive tunneling mechanism [26]. In order to obtain the temperature and energy dependence of $\beta$, the measured decay curves were fitted by Eq. (3) through plotting $\ln [\ln \times$ $\left.I_{0} / I(t)\right]$ vs $\ln$ (time), using $\beta$ and $\tau$ as fitting parameters. The result for the PL decay of the peak energy emission at $14 \mathrm{~K}$ is given in Fig. 2(b). The fitted $\beta$ is 0.84 and $\tau$ is $212 \mathrm{ps.} \mathrm{In}$ Fig. 3, we have plotted the temperature (a) and energy (b) dependence of $\beta$ and found that $\beta$ almost remains unchanged in both cases. This observed temperature and energy independence of $\beta$ is consistent with topological disorder model [26]. It is noted that our $\beta$ values are larger than the reported value of $\beta$ in porous silicon [24]. It reflects a less degree of disorder in our AlInGaN epilayers compared to porous silicon.

In order to further understand the dimensional property of the localization centers, we have studied radiative and non-radiative recombination dynamics by combining the PL
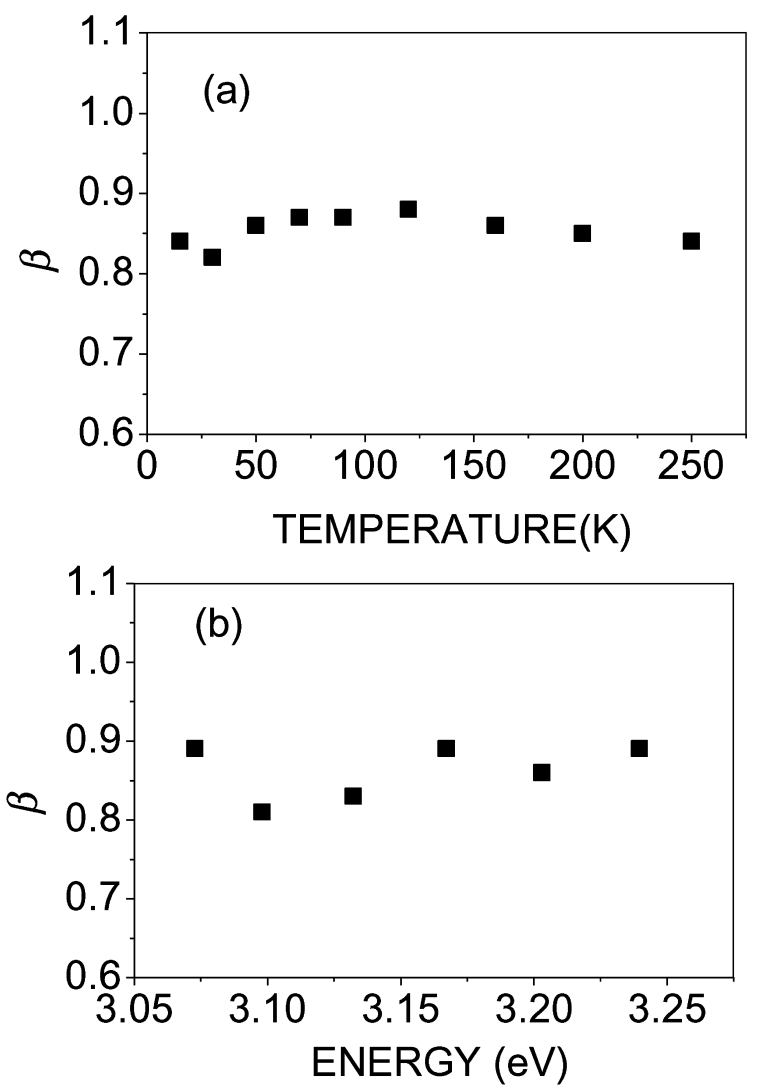

Fig. 3. $\beta$ as a function of the temperature (a), and emission energy at $250 \mathrm{~K}(\mathrm{~b})$. 
and TRPL results. The values of the radiative lifetime $\tau_{\text {rad }}$ and non-radiative lifetime $\tau_{\text {nonrad }}$ are deduced from the longer component of PL decay ( $\left.\tau_{\mathrm{PL}}\right)$ and the PL intensity as a function of temperature using the relation $\eta_{\text {int }}=$ $1 /\left(1+\tau_{\text {rad }} / \tau_{\text {nonrad }}\right)$, where $\eta_{\text {int }}$ is an internal quantum efficiency. To simplify, $\eta_{\text {int }}$ is set to unity at low temperature since the non-radiative recombination process is generally frozen at low temperature, as demonstrated in the inset of Fig. 4. Then, $\tau_{\text {rad }}$ and $\tau_{\text {nonrad }}$ can be expressed as

$\tau_{\mathrm{rad}}(T)=\frac{I(14 \mathrm{~K})}{I(T)} \tau(T)$

$\tau_{\text {nonrad }}(T)=\frac{I(14 \mathrm{~K})}{I(14 \mathrm{~K})-I(T)} \tau(T)$.

In Fig. 4 , the deduced $\tau_{\text {rad }}$ and $\tau_{\text {nonrad }}$ are plotted as a function of temperature. It can be seen that there is not much variation of $\tau_{\text {rad }}$ from 14 to $250 \mathrm{~K}$. It is a typical characteristic of the 0D excitons. Therefore it is believed that the emission of our sample originates from the excitons localized in disordered QD-like nanostructures up to $250 \mathrm{~K}$.

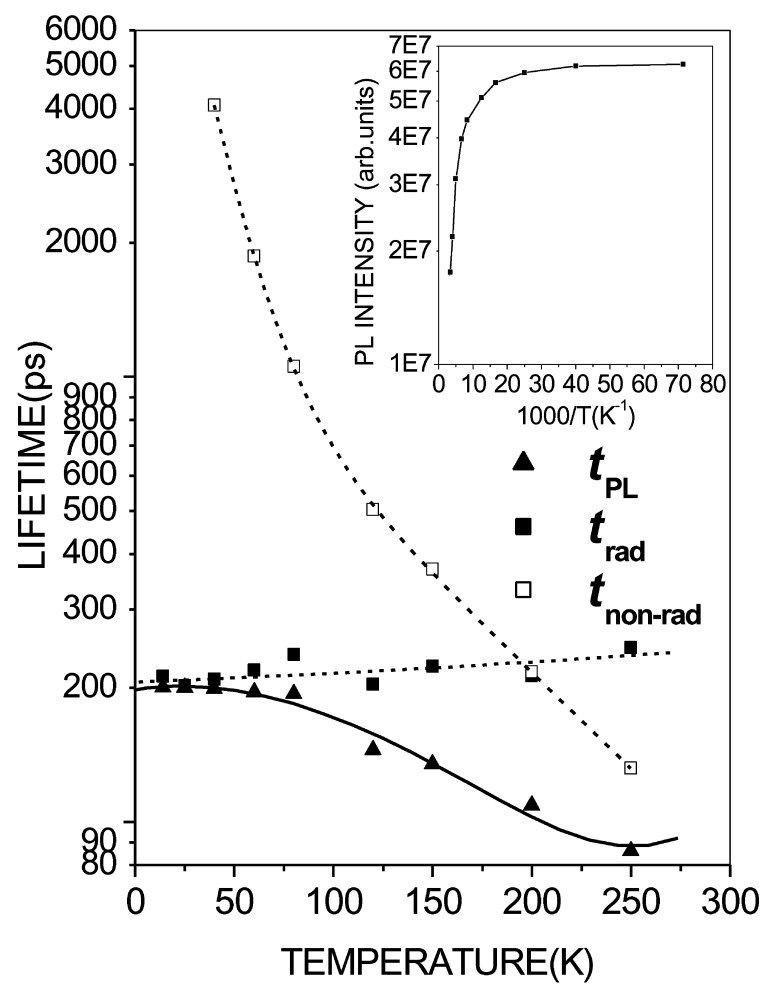

Fig. 4. Temperature dependence of PL lifetime $\left(\tau_{\mathrm{PL}}\right)$, radiative and non-radiative lifetimes ( $\tau_{\text {rad }}$ and $\left.\tau_{\text {non-rad }}\right)$ deduced from the temperature-dependent TRPL and PL data. The inset is the integrated PL intensity at different temperature, showing that the radiative recombination dominates the PL emission at low temperature.

\section{Conclusion}

In conclusion, we have measured temperature-dependent PL and TRPL to study the carrier recombination dynamics in AlInGaN alloys. Unusual temperature dependence of PL peak energy is observed and is well fitted by the thermal activation and transfer model. This result provides evidence for the exciton localization in the QD-like potentials in our AlInGaN alloy. The TRPL signals are found to be well fitted as a stretched exponential decay from 14 to $250 \mathrm{~K}$, suggesting that the spontaneous emission is due to the radiative recombination of carriers localized in a disordered system. The disorder is attributed to a randomly distributed quantum nanostructures such as quantum dots or clusters caused by indium fluctuations, as in the case of InGaN alloy. By studying the dependence of the dispersive exponent $\beta$ on the temperature and emission energy, we suggest that the hopping of excitons among localized states dominates the carrier diffusion. The localized states are considered to have OD density of states up to $250 \mathrm{~K}$.

\section{Acknowledgements}

This work was supported by the special funds for Major State Basic Research Project No. G001CB3095 and No. G2000683 of China, Grant No. 19974045 from NSFC, Grant No. 10274081 from NSFC, Nanotech Project of CAS and Grant HKUST 6125/98P.

\section{References}

[1] M. Asif Khan, J.W. Yang, G. Simin, R. Gaska, M.S. Shur, H. Zur Loye, Appl. Phys. Lett. 76 (2000) 1161.

[2] M. Asif Khan, J.W. Yang, G. Simin, R. Gaska, M.S. Shur, A.D. Bykhovski, Appl. Phys. Lett. 75 (1999) 2806.

[3] M.E. Aumer, S.F. LeBoef, S.M. Bedair, M. Smith, J.Y. Lin, H.X. Jiang, Appl. Phys. Lett. 77 (2000) 821.

[4] M.E. Aumer, S.F. LeBoeuf, F.G. Mclntosh, S.M. Bedair, Appl. Phys. Lett. 69 (2000) 821.

[5] J. Zhang, J. Yang, G. Simin, M. Shatalov, M. Asif Khan, Appl. Phys. Lett. 77 (2000) 2668.

[6] V. Adivarahan, A. Chitnis, J.P. Zhang, M. Shatalov, J.W. Yang, G. Simin, M. Asif Khan, Appl. Phys. Lett. 79 (2001) 4240 .

[7] J.P. Zhang, V. Adivarahan, H.M. Wang, Q. Fareed, E. Kuokstis, A. Chitnis, M. Shatalov, Jpn J. Appl. Phys. Part 2 40 (2001) L921.

[8] J. Zhang, E. Kuoksitis, Q. Fareed, H. Wang, J. Yang, G. Simin, M. Asif Khan, R. Gaska, M. Shur, Appl. Phys. Lett. 79 (2001) 925.

[9] G. Tamulaitis, K. Kazlauskas, S. Jursenas, A. Zukauskas, Appl. Phys. Lett. 77 (2000) 2136.

[10] M.-Y. Ryu, C.Q. Chen, E. Kuokstis, J.W. Yang, G. Simin, M. Asif Khan, Appl. Phys. Lett. 80 (2002) 3730.

[11] M.-Y. Ryu, C.Q. Chen, E. Kuokstis, J.W. Yang, G. Simin, M. Asif Khan, Appl. Phys. Lett. 80 (2002) 3943. 
[12] J.S. Huang, X. Dong, X.D. Luo, D.B. Li, X.L. Liu, Z.Y. Xu, W.K. Ge, J. Cryst. Growth 247 (2003) 84.

[13] W. Shan, T.J. Schmidt, X.H. Yang, S.J. Hwang, J.J. Song, B. Goldenberg, Appl. Phys. Lett. 66 (1995) 985.

[14] W. Shan, B.D. Little, J.J. Song, Z.C. Feng, M. Schurman, R.A. Stall, Appl. Phys. Lett. 69 (1996) 3315.

[15] Z.Y. Xu, Z.D. Lu, X.P. Yang, Z.L. Yuan, B.Z. Zheng, J.Z. Xu, Phys. Rev. B 54 (16) (1996) 11528.

[16] J. Li, B. Nam, K.H. Kim, J.Y. Lin, H.X. Jiang, Appl. Phys. Lett. 78 (2001) 61

[17] K.P. O’Donnell, R.W. Martin, P.G. Middleton, Phys. Rev. Lett. 82 (1999) 237.

[18] V. Lemos, E. Silveira, J.R. Leite, A. Tabata, R. Trentin, L.M.R. Scolfaro, Phys. Rev. Lett. 84 (2000) 3666.
[19] Q. Li, S.J. Xu, W.C. Cheng, M.H. Xie, S.Y. Tong, Appl. Phys. Lett. 79 (2001) 1810.

[20] Z.Y. Xu, Z.D. Lu, Z.L. Yuan, X.P. Yand, B.Z. Zheng, J.Z. Xu, Superlattices Microstruct. 23 (1998) 381.

[21] X. Chen, B. Henderson, K.P. O’Donnell, Appl. Phys. Lett. 60 (1992) 2672.

[22] M. Pophristic, F.H. Long, Appl. Phys. Lett. 73 (1998) 3550.

[23] S.F. Chichibu, M. Sugiyama, T. Onuma, Appl. Phys. Lett. 79 (2001) 4319.

[24] L. Pavesi, M. Ceschini, Phys. Rev. B 48 (1993) 17625.

[25] A.Yu. Kobitski, K.S. Zhuravlev, Phys. Rev. B 63 (2001) 115423.

[26] J. Kakalios, R.A. Street, W.B. Jackson, Phys. Rev. Lett. 59 (9) (1987) 1037. 